\title{
SYMMETRY MATTERS FOR SIZES OF EXTENDED FORMULATIONS
}

\author{
VOLKER KAIBEL, KANSTANTSIN PASHKOVICH, AND DIRK OLIVER THEIS
}

\begin{abstract}
In 1991, Yannakakis [18] proved that no symmetric extended formulation for the matching polytope of the complete graph $K_{n}$ with $n$ nodes has a number of variables and constraints that is bounded subexponentially in $n$. Here, symmetric means that the formulation remains invariant under all permutations of the nodes of $K_{n}$. It was also conjectured in [18] that "asymmetry does not help much," but no corresponding result for general extended formulations has been found so far. In this paper we show that for the polytopes associated with the matchings in $K_{n}$ with $\lfloor\log n\rfloor$ edges there are non-symmetric extended formulations of polynomial size, while nevertheless no symmetric extended formulations of polynomial size exist. We furthermore prove similar statements for the polytopes associated with cycles of length $\lfloor\log n\rfloor$. Thus, with respect to the question for smallest possible extended formulations, in general symmetry requirements may matter a lot. Compared to the extended abstract [13], this paper does not only contain proofs that had been ommitted there, but it also presents slightly generalized and sharpened lower bounds.
\end{abstract}

\section{INTRODUCTION}

Linear Programming techniques have proven to be extremely fruitful for combinatorial optimization problems with respect to both structural analysis and the design of algorithms. In this context, the paradigm is to represent the problem by a polytope $P \subseteq \mathbb{R}^{m}$ whose vertices correspond to the feasible solutions of the problem in such a way that the objective function can be expressed by a linear functional $x \mapsto\langle c, x\rangle$ on $\mathbb{R}^{m}$ (with some $c \in \mathbb{R}^{m}$ ). If one succeeds in finding a description of $P$ by means of linear constraints, then algorithms as well as structural results from Linear Programming can be exploited. In many cases, however, the polytope $P$ has exponentially (in $m$ ) many facets, thus $P$ can only be described by exponentially many inequalities. Also it may be that the inequalities needed to describe $P$ are too complicated to be identified.

Date: June 26, 2021.

Research by K. Pashkovich has been supported by the International Max Planck Research School for Analysis, Design and Optimization in Chemical and Biochemical Process Engineering Magdeburg

An extended abstract of this work has been published as [13]: V. Kaibel, K. Pashkovich, and D. O. Theis, Symmetry matters for the sizes of extended formulations, in: F. Eisenbrand and B. Shepherd (eds.), Integer Programming and Combinatorial Optimization (Proc. IPCO XIV), volume 6080 of LNCS, pages 135-148. Springer, 2010. 
In some of these cases one may find an extended formulation for $P$, i.e., a (preferably small and simple) description by linear constraints of another polyhedron $Q \subseteq \mathbb{R}^{d}$ in some higher dimensional space that projects to $P$ via some (simple) affine map $p: \mathbb{R}^{d} \rightarrow \mathbb{R}^{m}$ with $p(y)=T y+t$ for all $y \in \mathbb{R}^{d}$ (and some $T \in \mathbb{R}^{m \times d}, t \in \mathbb{R}^{m}$ ). As we have $\max \{\langle c, x\rangle: x \in P\}=\max \left\{\left\langle T^{\mathrm{t}} c, y\right\rangle:\right.$ $y \in Q\}+\langle c, t\rangle$ for each $c \in \mathbb{R}^{m}$, one can solve linear optimization problems over $P$ by solving linear optimization problems over $Q$ in this case.

As for a guiding example, let us consider the spanning tree polytope

$$
\mathrm{P}_{\text {spt }}(n)=\operatorname{conv}\left\{\chi(T) \in\{0,1\}^{E_{n}}: T \subseteq E_{n} \text { spanning tree of } K_{n}\right\},
$$

where $K_{n}=\left([n], E_{n}\right)$ denotes the complete graph with node set $[n]=\{1, \ldots, n\}$ and edge set $E_{n}=\{\{v, w\}: v, w \in[n], v \neq w\}$, and $\chi(A) \in\{0,1\}^{B}$ is the characteristic vector of the subset $A \subseteq B$ of $B$, i.e., for all $b \in B$, we have $\chi(A)_{b}=1$ if and only if $b \in A$. Thus, $\mathrm{P}_{\text {spt }}(n)$ is the polytope associated with the bases of the graphical matroid of $K_{n}$, and we have (see [6])

$$
\begin{aligned}
& \mathrm{P}_{\text {spt }}(n)=\left\{x \in \mathbb{R}_{+}^{E_{n}}: x\left(E_{n}\right)=n-1,\right. \\
&x(E(S)) \leq|S|-1 \text { for all } S \subseteq[n], 2 \leq|S| \leq n-1\},
\end{aligned}
$$

where $\mathbb{R}_{+}^{E_{n}}$ is the nonnegative orthant of $\mathbb{R}_{n}^{E}$, we denote by $E(S)$ the subset of all edges with both nodes in $S$, and $x(F)=\sum_{e \in F} x_{e}$ for $F \subseteq E_{n}$. This linear description of $\mathrm{P}_{\text {spt }}(n)$ has an exponential (in $n$ ) number of constraints, and as all the inequalities define pairwise different facets, none of them is redundant.

The following much smaller extended formulation for $\mathrm{P}_{\mathrm{spt}}(n)$ (with $O\left(n^{3}\right)$ variables and constraints) appears in [4] (and a similar one in [18], where it is attributed to [14]). Let us introduce additional variables $z_{e, v, u}$ for all $e \in E_{n}, v \in e$, and $u \in[n] \backslash e$. While each spanning tree $T \subseteq E_{n}$ is represented by its characteristic vector $x^{(T)}=\chi(T)$ in $\mathrm{P}_{\mathrm{spt}}(n)$, in the extended formulation it will be represented by the vector $y^{(T)}=\left(x^{(T)}, z^{(T)}\right)$ with $z_{e, v, u}^{(T)}=1$ (for $e \in E_{n}, v \in e, u \in[n] \backslash e$ ) if $e \in T$ and $u$ is contained in the component of $v$ in $T \backslash e$, and with $z_{e, v, u}^{(T)}=0$ otherwise.

The polyhedron $Q_{\mathrm{spt}}(n) \subseteq \mathbb{R}^{d}$ defined by the nonnegativity constraints $x \geq \mathbf{0}$, $z \geq \mathbf{0}$, the equations $x\left(E_{n}\right)=n-1$,

$$
x_{\{v, w\}}-z_{\{v, w\}, v, u}-z_{\{v, w\}, w, u}=0 \text { for all pairwise distinct } v, w, u \in[n],
$$

as well as

$$
x_{\{v, w\}}+\sum_{u \in[n] \backslash\{v, w\}} z_{\{v, u\}, u, w}=1 \quad \text { for all distinct } v, w \in[n],
$$

satisfies $p\left(Q_{\mathrm{spt}}(n)\right)=\mathrm{P}_{\mathrm{spt}}(n)$, where $p: \mathbb{R}^{d} \rightarrow \mathbb{R}_{n}^{E}$ is the orthogonal projection onto the $x$-variables. This follows from observing that, for each spanning tree $T \subseteq E_{n}$, the vector $y^{(T)}=\left(x^{(T)}, z^{(T)}\right)$ satisfies (2) and (3), and on the other hand, every nonnegative vector $y=(x, z) \in \mathbb{R}_{+}^{d}$ satisfying (2) and (3) also satisfies $x(E(S)) \leq|S|-1$ for all $S \subseteq[n]$ with $|S| \geq 2$. Indeed, to see the latter claim, 
one adds equations (2) for all pairwise distinct $v, w, u \in S$ in order to obtain (after division by two and renaming summation indices)

$$
(|S|-2) x(E(S))=\sum_{v, w \in S, v \neq w} \sum_{u \in S \backslash\{v, w\}} z_{\{v, u\}, u, w},
$$

where, due to (3) and $z \geq \mathbf{0}$, the right-hand side is bounded from above by

$$
\sum_{v, w \in S, v \neq w}\left(1-x_{\{v, w\}}\right)=|S|(|S|-1)-2 x(E(S)),
$$

which together with (4) implies $x(E(S)) \leq|S|-1$.

For many other polytopes (with exponentially many facets) associated with polynomial time solvable combinatorial optimization problems polynomially sized extended formulations can be constructed as well (see, e.g., the recent survey [4]). Probably the most prominent problem in this class for which, however, no such small formulation is known, is the matching problem. In fact, Yannakakis [18] proved that no symmetric polynomially sized extended formulation of the matching polytope exists.

Here, symmetric refers to the symmetric group $\mathfrak{S}(n)$ of all permutations $\pi$ : $[n] \rightarrow[n]$ of the node set $[n]$ of $K_{n}$ acting on $E_{n}$ via ${ }^{1} \pi \cdot\{v, w\}=\{\pi(v), \pi(w)\}$ for all $\pi \in \mathfrak{S}(n)$ and $\{v, w\} \in E_{n}$. Clearly, this action of $\mathfrak{S}(n)$ on $E_{n}$ induces an action on the set of all subsets of $E_{n}$. For instance, this yields an action on the spanning trees of $K_{n}$, and thus, on (the vertices of) $\mathrm{P}_{\text {spt }}(n)$. The extended formulation of $\mathrm{P}_{\mathrm{spt}}(n)$ discussed above is symmetric in the sense that, for every $\pi \in \mathfrak{S}(n)$, replacing all indices associated with edges $e \in E_{n}$ and nodes $v \in[n]$ by $\pi . e$ and $\pi . v$, respectively, does not change the set of constraints in the formulation. Phrased informally, all subsets of nodes of $K_{n}$ of equal cardinality play the same role in the formulation. For a general definition of symmetric extended formulations see Section 2

In order to describe the main results of Yannakakis' paper [18] and the contributions of the present paper, let us denote by

$$
\mathcal{M}^{\ell}(n)=\left\{M \subseteq E_{n}: M \text { matching in } K_{n},|M|=\ell\right\}
$$

the set of all matchings of size $\ell$ (a matching being a subset of edges no two of which share a node), and by

$$
\mathrm{P}_{\text {match }}^{\ell}(n)=\operatorname{conv}\left\{\chi(M) \in\{0,1\}^{E_{n}}: M \in \mathcal{M}^{\ell}(n)\right\}
$$

the associated polytope. According to Edmonds [5] the perfect matching polytope $\mathrm{P}_{\text {match }}^{n / 2}(n)$ (for even $n$ ) is described by

$$
\begin{aligned}
& \mathrm{P}_{\text {match }}^{n / 2}(n)=\left\{x \in \mathbb{R}_{+}^{E_{n}}: x(\delta(v))=1 \text { for all } v \in[n],\right. \\
& \qquad x(\delta(S)) \geq 1 \text { for all } S \subseteq[n], 3 \leq|S| \text { odd }\}
\end{aligned}
$$

\footnotetext{
${ }^{1}$ For an action $G \times E \rightarrow E$ of a group $G$ on a set $E$ we use the notation $g . e$ for the image of $(g, e)$ under the action, since it makes many formulas easier to read.
} 
(with $\delta(S)=\left\{e \in E_{n}:|e \cap S|=1\right\}$ and $\delta(v)=\delta(\{v\})$ ). Yannakakis [18, Thm. 1 and its proof] shows that there is a constant $C>0$ such that, for every extended formulation for $\mathrm{P}_{\text {match }}^{n / 2}(n)$ (with $n$ even) that is symmetric in the sense above, the number of variables and constraints is at least $C \cdot\left(\begin{array}{c}n \\ \lfloor n / 4\rfloor\end{array}\right)=2^{\Omega(n)}$. This in particular implies that there is no polynomial size symmetric extended formulation for the matching polytope of $K_{n}$ (the convex hulls of characteristic vectors of all matchings in $K_{n}$ ) of which the perfect matching polytope is a face.

Yannakakis [18] moreover obtains a similar (maybe less surprising) result for traveling salesman polytopes. Denoting by

$$
\mathcal{C}^{\ell}(n)=\left\{C \subseteq E_{n}: C \text { cycle in } K_{n},|C|=\ell\right\}
$$

the set of all (simple) cycles of length $\ell$ in $K_{n}$, and by

$$
\mathrm{P}_{\text {cycl }}^{\ell}(n)=\operatorname{conv}\left\{\chi(C) \in\{0,1\}^{E_{n}}: C \in \mathcal{C}^{\ell}(n)\right\}
$$

the associated polytopes, the traveling salesman polytope is $\mathrm{P}_{\text {cycl }}^{n}(n)$. Suitably identifying $\mathrm{P}_{\text {match }}^{n / 2}(n)$ (for even $n$ ) with a face of $\mathrm{P}_{\text {cycl }}^{3 n}(3 n)$, Yannakakis concludes that all symmetric extended formulations for $\mathrm{P}_{\text {cycl }}^{n}(n)$ have size at least $2^{\Omega(n)}$ as well [18, Thm. 2 and its proof].

Yannakakis' results in a fascinating way illuminate the borders of our principal abilities to express combinatorial optimization problems like the matching or the traveling salesman problem by means of linear constraints. However, they only refer to linear descriptions that respect the inherent symmetries in the problems. In fact, the second open problem mentioned in the concluding section of [18] is described as follows: "We do not think that asymmetry helps much. Thus, prove that the matching and TSP polytopes cannot be expressed by polynomial size LP's without the asymmetry assumption."

Indeed, it was shown very recently (and while this paper was under review) that the traveling salesman polytope does also not possess any non-symmetric compact extended formulation [8]. The correpsonding question concerning the matching polytope, however, still remains open.

The contribution of our paper is to show that, in contrast to the assumption expressed in the quotation above, asymmetry can help much, or, phrased differently, that symmetry requirements on extended formulations indeed can matter significantly with respect to the minimal sizes of extended formulations. Our main results are that both $\mathrm{P}_{\text {match }}^{\lfloor\log n\rfloor}(n)$ and $\mathrm{P}_{\operatorname{cycl}}^{\lfloor\log n\rfloor}(n)$ do not admit symmetric extended formulations of polynomial size, while they have non-symmetric extended formulations of polynomial size (see Cor. 18 and 23 for matchings, as well as Cor. 27 and 29 for cycles). The corresponding theorems from which these corollaries are derived provide some more general and more precise results for $\mathrm{P}_{\text {match }}^{\ell}(n)$ and $\mathrm{P}_{\text {cycl }}^{\ell}(n)$. In order to establish the lower bounds for symmetric extensions, we adapt the techniques developed by Yannakakis [18]. The constructions of the compact non-symmetric extended formulations rely on small families of perfect hash functions [1, 9, 16]. 
The paper is organized as follows. In Section 2, we provide definitions of extensions, extended formulations, their sizes, the crucial notion of a section of an extension, symmetry of an extension, and we give some auxilliary results. In Section 3 we derive (using ideas from [18]) lower bounds on the sizes of symmetric extended formulations for the polytopes $\mathrm{P}_{\text {match }}^{\ell}(n)$ associated with cardinality restricted matchings. In Section 4, we then describe our non-symmetric extended formulations for these polytopes. Finally, in Section 5 we present the results on $\mathrm{P}_{\text {cycl }}^{\ell}(n)$. Some remarks conclude the paper in Section 6

An extended abstract [13] of this work has appeared in the proceedings of IPCO XIV. The present paper does not only contain additional proofs that have been omitted in [13] and a simplified and, as we believe, clearer presentation of the proof of Theorem 15, but it also slightly sharpens our lower bound results in two ways (based on the new Lemma 10): We now prove lower bounds on the mere number of inequalities (rather than on the number of inequalities plus the number of variables) of symmetric extended formulations, and these results now refer to the more general notion of symmetry obtained from considering arbitrary isometries instead of coordinate permutations only.

Acknowledgements. We thank Christian Bey for discussions on invariant subspaces and the referees for their comments, in particular for pointing out the simple probabilistic argument for the existence of small families of perfect hash functions (Theorem 24).

\section{EXTENDED Formulations, EXTENSIONS, AND SyMmETRY}

Here, we formalize the central notions used in this paper and establish some basic results we will rely on later.

Definition 1. An extension of a polytope $P \subseteq \mathbb{R}^{m}$ is a polyhedron $Q \subseteq \mathbb{R}^{d}$ together with an affine projection $p: \mathbb{R}^{d} \rightarrow \mathbb{R}^{m}$ with $p(Q)=P$. The size of an extension is the number of its facets.

Definition 2. An extension $Q \subseteq \mathbb{R}^{d}, p: \mathbb{R}^{d} \rightarrow \mathbb{R}^{m}$ of a polytope $P \subseteq \mathbb{R}^{m}$ is called a subspace extension if $Q$ is the intersection of an affine subspace of $\mathbb{R}^{d}$ and the nonnegative orthant $\mathbb{R}_{+}^{d}$.

For instance, the polyhedron $Q_{\mathrm{spt}}(n)$ defined in the Introduction is a subspace extension of the spanning tree polytope $\mathrm{P}_{\mathrm{spt}}(n)$.

Definition 3. A (finite) system of linear equations and inequalities whose set of solutions (together with some projection) forms an extension of $P$ is an extended formulation for $P$. The size of an extended formulation is its number of inequalities (including nonnegativity constraints, but not equations).

Clearly, the size of an extended formulation is at least as large as the size of the extension it describes. Conversely, every extension is described by an extended formulation of at most its size.

Extensions or extended formulations of a family of polytopes $P \subseteq \mathbb{R}^{m}$ (for varying $m$ ) are compact if their sizes and the encoding lengths of the coefficients 
needed to describe them can be bounded by a polynomial in $m$ and the maximal encoding length of all components of all vertices of $P$. Clearly, the extension $Q_{\mathrm{spt}}(n)$ of $\mathrm{P}_{\mathrm{spt}}(n)$ from the Introduction is compact.

Definition 4. For an extension $Q \subseteq \mathbb{R}^{d}, p: \mathbb{R}^{d} \rightarrow \mathbb{R}^{m}$ of a polytope $P \subseteq \mathbb{R}^{m}$, the fiber of $x \in P$ is the set $p^{-1}(x)=\left\{y \in \mathbb{R}^{d}: p(y)=x\right\}$.

Definition 5. For an extension $Q \subseteq \mathbb{R}^{d}, p: \mathbb{R}^{d} \rightarrow \mathbb{R}^{m}$ of a polytope $P \subseteq \mathbb{R}^{m}$, a section $s: X \rightarrow Q$ is a map that assigns to every vertex $x$ of $P$ some point $s(x) \in Q \cap p^{-1}(x)$ in the intersection of the extension $Q$ and the fiber $p^{-1}(x)$.

Such a section induces a bijection between $X$ and its image $s(X) \subseteq Q$, whose inverse is given by $p$. In the spanning tree example from the Introduction, the assignment $\chi(T) \mapsto y^{(T)}=\left(x^{(T)}, z^{(T)}\right)$ defined such a section. Note that, in general, sections will not be induced by affine maps. In fact, if a section is induced by an affine map $s: \mathbb{R}^{m} \rightarrow \mathbb{R}^{d}$, then the intersection of $Q$ with the affine subspace of $\mathbb{R}^{d}$ generated by $s(X)$ is isomorphic to $P$, thus $Q$ has at least as many facets as $P$.

If $s: X \rightarrow Q$ is a section for some extended formulation of $P$ then, for each inequality $\langle c, y\rangle \leq \gamma$ in the formulation, we call the vector in $\mathbb{R}_{+}^{X}$ with entries $\gamma-\langle c, s(x)\rangle(x \in X)$ a section slack covector. Similarly, any valid inequality $\langle a, x\rangle \leq \beta$ for $P$ defines a slack covector in $\mathbb{R}_{+}^{X}$ with entries $\beta-\langle a, x\rangle(x \in X)$. One finds that every slack covector is a conic combination of the section slack covectors [7, Cor. 2.5]. In particular, we derive the following proposition (via the trivial direction of the Farkas-Lemma), which follows from the fact that every subspace extension can be described by a linear system containing linear equations and non-negativity constraints only, implying that in this case the coordinates of the section slack covectors simply correspond to the values of the section coordinate functions.

Proposition 6. If $s: X \rightarrow Q$ is a section for a subspace extension $Q \subseteq \mathbb{R}_{+}^{d}$ of $P=\operatorname{conv}(X)$ and $\langle a, x\rangle \leq \beta$ is valid for $P$ then the system

$$
\begin{aligned}
\sum_{x \in X} s_{j}(x) \cdot \lambda_{x} & \geq 0 \text { for all } j \in[d] \\
\sum_{x \in X}(\beta-\langle a, x\rangle) \cdot \lambda_{x} & =-1
\end{aligned}
$$

does not have any solution $\lambda \in \mathbb{R}^{X}$.

Aiming to prove that a (subspace) extension of a certain type does not exist one thus can first construct some appropriate section for such an extension for which one then exhibits, for some inequality valid for $P$, a solution to (6), (7).

In order to define the notion of symmetry of an extension precisely, we will deal with groups of affine transformations $\pi: \mathbb{R}^{m} \rightarrow \mathbb{R}^{m}$. We will frequently use the notation $\pi . x=\pi(x)$ for $x \in \mathbb{R}^{m}$ and $\pi . S=\pi(S)$ for $S \subseteq \mathbb{R}^{m}$. Let us denote by $\mathcal{O}(d)$ the group of all affine isometries of $\mathbb{R}^{d}$, i.e., the set of all maps $\varkappa: \mathbb{R}^{d} \rightarrow \mathbb{R}^{d}$ of the form $\varkappa(y)=U y+u$ with an orthogonal matrix $U \in \mathbb{R}^{d \times d}$ (i.e., $U U^{\mathrm{t}}=\mathbf{I}$ ) 
and $u \in \mathbb{R}^{d}$. The group $\mathfrak{S}(d)$ of all bijective maps from $[d]=\{1, \ldots, d\}$ to itself acts on $\mathbb{R}^{d}$ by coordinate permutations via $(\sigma . y)_{j}=y_{\sigma^{-1}(j)}$ for all $y \in \mathbb{R}^{d}$, $\sigma \in \mathfrak{S}(d)$, and $j \in[d]$. Identifying $\sigma \in \mathfrak{S}(d)$ with the isometry defined via $y \mapsto \sigma . y$, we consider $\mathfrak{S}(d)$ as a subgroup of $\mathcal{O}(d)$.

Suppose that $P \subseteq \mathbb{R}^{m}$ is a polytope with vertex set $X \subseteq \mathbb{R}^{m}$, and $G$ is a group of affine transformations $\pi: \mathbb{R}^{m} \rightarrow \mathbb{R}^{m}$ with $\pi \cdot P=P$. Clearly, every $\pi \in G$ permutes the vertices of $P$. Usually, $G$ will be a subgroup of coordinate permutations of $\mathbb{R}^{m}$, i.e., a subgroup of $\mathfrak{S}(m)$.

Definition 7. An extension $Q \subseteq \mathbb{R}^{d}$ of $P$ with projection $p: \mathbb{R}^{d} \rightarrow \mathbb{R}^{m}$ is isometry-symmetric with respect to $G$ (or, for short, symmetric), if for every $\pi \in G$ there is an isometry $\varkappa_{\pi} \in \mathcal{O}(d)$ with $\varkappa_{\pi} \cdot Q=Q$ and

$$
p\left(\varkappa_{\pi} \cdot y\right)=\pi \cdot p(y) \quad \text { for all } y \in Q
$$

(see Fig. 11); the extension is called coordinate-symmetric if all these $\varkappa_{\pi}$ can be chosen to be from $\mathfrak{S}(d)$.
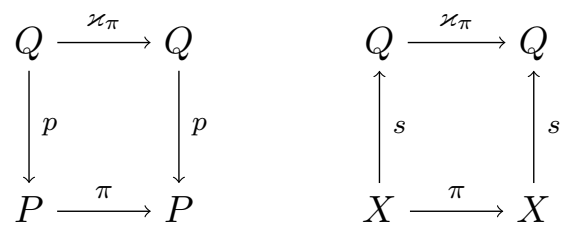

Figure 1. Relations (8) (left) and 20) (right) from the definitions of symmetry and weak coordinate-symmetry, respectively.

The prime examples of symmetric extensions arise from extended formulations that "look symmetric".

Definition 8. An extended formulation $A^{=} y=b^{=}, A \leq y \leq b \leq$ describing the polyhedron

$$
Q=\left\{y \in \mathbb{R}^{d}: A^{=} y=b^{=}, A^{\leq} y \leq b^{\leq}\right\}
$$

extending $P \subseteq \mathbb{R}^{m}$ as above is symmetric (with respect to the action of $G$ on $P$ ), if for every $\pi \in G$ there is some $\varkappa_{\pi} \in \mathfrak{S}(d)$ satisfying (8) and there are two permutations $\varrho_{\pi}^{=}$and $\varrho_{\pi}^{\leq}$of the rows of $\left(A^{=}, b^{=}\right)$and $\left(A^{\leq}, b^{\leq}\right)$, respectively, such that the corresponding simultaneous permutations of the columns and the rows of the matrices $\left(A^{=}, b^{=}\right)$and $\left(A^{\leq}, b^{\leq}\right)$leave them unchanged.

Clearly, in this situation the permutations $\varkappa_{\pi}$ satisfy $\varkappa_{\pi} \cdot Q=Q$, which implies the following.

Lemma 9. Every symmetric extended formulation defines a (coordinate-)symmetric extension.

One example of a symmetric extended formulation is the extended formulation for the spanning tree polytope described in the Introduction (with respect to the group $G$ of all permutations of the nodes of the complete graph). 
The following lemma shows that we can restrict our attention to coordinatesymmetric subspace extensions when searching for the minimum size of any (isometry-)symmetric extension of a given polytope $P$. In particular, the minimum size of a symmetric extension of a polytope is attained by a pointed symmetric extension.

Lemma 10. If a polytope has an isometry-symmetric extension of size $f$, then it has also a coordinate-symmetric subspace extension of size $f$.

Proof. Let $P \subseteq \mathbb{R}^{m}$ be some polytope, $Q \subseteq \mathbb{R}^{d}$ some polyhedron with $f$ facets, $p: \mathbb{R}^{d} \rightarrow \mathbb{R}^{m}$ some affine projection with $p(Q)=P$, and $G$ some group acting on $P$ and the set $X$ of vertices of $P$ such that, for every $\pi \in G$, there is some $\varkappa_{\pi} \in \mathcal{O}(d)$ with $\varkappa_{\pi} \cdot Q=Q$ and $p\left(\varkappa_{\pi} \cdot y\right)=\pi \cdot p(y)$ for all $y \in Q$.

We denote the affine hull of $Q$ by aff $(Q)$ and by $L \subseteq \mathbb{R}^{d}$ the linear subspace parallel to aff $(Q)$. The polyhedron $Q$ has a unique (up to reordering of the inequalities) description

$$
Q=\left\{y \in \mathbb{R}^{d}: y \in \operatorname{aff}(Q), A y \leq b\right\}
$$

with $A \in \mathbb{R}^{f \times d}$ and $b \in \mathbb{R}^{f}$, if we require, for each $i \in[f]$, that $A_{i, \star} \in L$ and $\left\|A_{i, \star}\right\|=1$ hold for the $i$-th row $A_{i, \star}$ of $A$. We define the affine map $\Delta^{Q}: \mathbb{R}^{d} \rightarrow$ $\mathbb{R}^{f}$ (the slack map of $Q$ ) via $\Delta^{Q}(y)=b-A y$ and call the image $\Delta(Q)=\Delta^{Q}(Q)$ of $Q$ under its slack map its slack representation. Note that $\Delta(Q)$ is the intersection of the nonnegative orthant $\mathbb{R}_{+}^{f}$ with the affine subspace $\Delta^{Q}(\operatorname{aff}(Q))$.

The lineality space lineal $(Q)=L \cap \operatorname{ker}(A)$ of $Q$ is the space of all directions of lines contained in $Q$. As $P=p(Q)$ is a polytope (thus bounded), we find

$$
p(y+r)=p(y) \quad \text { for all } y \in Q, r \in \operatorname{lineal}(Q) .
$$

The restriction $\Delta_{\perp}^{Q}$ of $\Delta^{Q}$ to the intersection of aff $(Q)$ with the orthogonal complement lineal $(Q)^{\perp}$ of the lineality space of $Q$ is a bijection

$$
\Delta_{\perp}^{Q}: \operatorname{aff}(Q) \cap \operatorname{lineal}(Q)^{\perp} \rightarrow \operatorname{aff}(\Delta(Q))
$$

with

$$
\left(\Delta_{\perp}^{Q}\right)^{-1}\left(\Delta^{Q}(y)\right)-y \in \operatorname{lineal}(Q) \text { for all } y \in Q,
$$

which in particular implies

$$
Q=\left(\Delta_{\perp}^{Q}\right)^{-1}(\Delta(Q))+\operatorname{lineal}(Q) .
$$

It suffices to prove that $\Delta(Q)$ is a coordinate-symmetric extension of $P$ via the affine projection $\tilde{p}=p \circ\left(\Delta_{\perp}^{Q}\right)^{-1}$ (which, of course, is defined on aff $(\Delta(Q)) \subseteq$ $\mathbb{R}^{f}$ only, but can be extended arbitrarily to $\mathbb{R}^{f}$ in order to formally satisfy the conditions of the definition of an extension). From (12) and (10) we deduce

$$
\tilde{p}(\Delta(Q))=p\left(\left(\Delta_{\perp}^{Q}\right)^{-1}(\Delta(Q))\right)=p(Q)=P .
$$

Therefore, we only need to exhibit, for each $\pi \in G$, some $\tilde{\varkappa}_{\pi} \in \mathfrak{S}(f)$ with

$$
\tilde{\varkappa}_{\pi} \cdot \Delta(Q)=\Delta(Q)
$$

and

$$
\tilde{p}\left(\tilde{\varkappa}_{\pi} \cdot z\right)=\pi \cdot \tilde{p}(z) \quad \text { for all } z \in \Delta(Q) .
$$


We construct $\tilde{\varkappa}_{\pi}$ from the map $\varkappa_{\pi} \in \mathcal{O}(d)$ with

$$
\varkappa_{\pi} \cdot Q=Q
$$

and

$$
p\left(\varkappa_{\pi} \cdot y\right)=\pi \cdot p(y) \quad \text { for all } y \in Q
$$

guaranteed to exist by the symmetry of the extension $Q$ of $P$. Let $U \in \mathbb{R}^{d \times d}$ be the orthogonal matrix and $u \in \mathbb{R}^{d}$ the vector with $\varkappa_{\pi} . y=U y+u$ for all $y \in \mathbb{R}^{d}$. From (15) (which implies aff $(Q)=\varkappa_{\pi}$. aff $(Q)$ ) and (9) we derive

$$
\begin{aligned}
Q=\varkappa_{\pi}^{-1} Q & =\left\{\varkappa_{\pi}^{-1} \cdot y: y \in \operatorname{aff}(Q), A y \leq b\right\} \\
& =\left\{y^{\prime} \in \mathbb{R}^{f}: \varkappa_{\pi} \cdot y^{\prime} \in \operatorname{aff}(Q), A\left(\varkappa_{\pi} \cdot y^{\prime}\right) \leq b\right\} \\
& =\left\{y^{\prime} \in \mathbb{R}^{f}: y^{\prime} \in \operatorname{aff}(Q),(A U) y^{\prime} \leq b-A u\right\} .
\end{aligned}
$$

Since $U$ is orthogonal and due to $\varkappa_{\pi}^{-1}$. aff $(Q)=\operatorname{aff}(Q)$ (implying $U^{\mathrm{t}} \ell \in L$ for all $\ell \in L$ ), the rows of the matrix $A U$ are contained in $L$ and have length one (since so do the rows of $A$ ). Thus, because of the uniqueness of the representation (9), there is a permutation $\sigma \in \mathfrak{S}(f)$ with

$$
(A U)_{i, \star}=A_{\sigma^{-1}(i), \star} \quad \text { and } \quad(b-A u)_{i}=b_{\sigma^{-1}(i)}
$$

for all $i \in[f]$. In order to show that $\tilde{\varkappa}_{\pi}=\sigma$ satisfies (13) and (14), we use the equation

$$
\Delta^{Q}\left(\varkappa_{\pi} \cdot y\right)=\sigma \cdot \Delta^{Q}(y)
$$

for all $y \in \mathbb{R}^{d}$, which follows readily from $\Delta^{Q}\left(\varkappa_{\pi} . y\right)=(b-A u)-(A U) y$, $\Delta^{Q}(y)=b-A y$, and (17).

For each $y \in Q$ equation (18) implies $\sigma . \Delta^{Q}(y) \in \Delta(Q)$ due to $\varkappa_{\pi} . y \in Q$. Thus we conclude $\sigma . \Delta(Q) \subseteq \Delta(Q)$, and hence $\sigma . \Delta(Q)=\Delta(Q)$ since $z \mapsto \sigma . z$ defines an isometry. Thus, (13) is established for $\tilde{\varkappa}_{\pi}=\sigma$. In order to also show (14) for this choice of $\tilde{\varkappa}_{\pi}$ it remains to prove

$$
\left.p\left(\left(\Delta_{\perp}^{Q}\right)^{-1}\left(\sigma \cdot \Delta^{Q}(y)\right)\right)=\pi \cdot p\left(\left(\Delta_{\perp}^{Q}\right)^{-1}\right)\left(\Delta^{Q}(y)\right)\right)
$$

for all $y \in Q$.

Due to (18), (15), and (11) the left-hand-side of (19) evaluates to $p\left(\varkappa_{\pi} \cdot y+r\right)$ for some $r \in \operatorname{lineal}(Q)$, and thus, due to $(10)$ and $(16)$, to $\pi . p(y)$. Similarly, the right-hand-side of (19) evaluates to $\pi \cdot p(y)$ as well, which concludes the proof.

The following lemma shows that coordinate-symmetric extensions have sections of a special type that will be crucial for the proof of the central result on the nonexistence of certain coordinate-symmetric subspace extensions (Theorem 15).

Definition 11. A section $s: X \rightarrow Q$ for an extension $Q$ of a polytope $P$ with vertex set $X$ and projection $p$ is called coordinate-symmetric if the action of $G$ on $s(X)$ induced by the action of the group $G$ on $X$ works by permutation of variables, i.e., if for every $\pi \in G$ there is a permutation $\varkappa_{\pi} \in \mathfrak{S}(d)$ with

$$
s(\pi \cdot x)=\varkappa_{\pi} \cdot s(x) \text { for all } x \in X
$$

(see Fig. 1). 
The following statement (and its proof) generalizes the construction of sections for coordinate-symmetric extensions of matching polytopes described in Yannakakis paper [18, Claim 1 in the proof of Thm. 1].

Lemma 12. Every coordinate-symmetric extension admits a coordinate-symmetric section.

Proof. Let us first observe that a coordinate-symmetric extension (with notations as above) satisfies

$$
\varkappa_{\pi} \cdot p^{-1}(x)=p^{-1}(\pi \cdot x) \quad \text { for all } \pi \in G \text { and } x \in X,
$$

(thus, $\varkappa_{\pi}$ permutes the fibers of points in $X$ according to $\pi$ ) since (8) readily implies $\varkappa_{\pi} \cdot p^{-1}(x) \subseteq p^{-1}(\pi . x)$, from which equality follows because both sets are affine subspaces of equal dimension (as all non-empty fibers of $p$ have the same dimension and $\varkappa_{\pi} \cdot p^{-1}(x)$ is an image of one of these fibers under a bijective affine transformation).

Let $\tilde{G}$ be the subgroup of $\mathfrak{S}(d)$ generated by $\left\{\varkappa_{\pi}: \pi \in G\right\}$. Clearly, we have

$$
\sigma \cdot Q=Q \quad \text { for all } \sigma \in \tilde{G} .
$$

We start the construction of a coordinate-symmetric section $s: X \rightarrow Q$ by choosing from each orbit $\{\sigma . x: \sigma \in G\}, x \in X$ under the action of $G$ some $x^{\star} \in X$ as well as an arbitrary point $y^{\star} \in Q \cap p^{-1}\left(x^{\star}\right)$ in the intersection of $Q$ and the fiber of $x^{\star}$. Actually, as we can consider the orbits one by one here, we will assume in the following that there is just one of them, i.e., the action of $G$ on $X$ is transitive. Denoting by

$$
\tilde{S}\left(x^{\star}\right)=\left\{\sigma \in \tilde{G}: \sigma \cdot p^{-1}\left(x^{\star}\right)=p^{-1}\left(x^{\star}\right)\right\},
$$

the subgroup of $\tilde{G}$ containing all permutations that map the fiber $p^{-1}\left(x^{\star}\right)$ to itself, we define

$$
s\left(x^{\star}\right)=\frac{1}{\left|\tilde{S}\left(x^{\star}\right)\right|} \sum_{\sigma \in \tilde{S}\left(x^{\star}\right)} \sigma \cdot y^{\star},
$$

which is a point in the convex set (polyhedron) $Q \cap p^{-1}\left(x^{\star}\right)$, because due to 22) we have $\sigma . y^{\star} \in Q \cap p^{-1}\left(x^{\star}\right)$ for all $\sigma \in \tilde{S}\left(x^{\star}\right)$. For each $x \in X$ we now choose some $\tau_{x} \in G$ with $\tau_{x} . x^{\star}=x$ (recall that we assumed the action of $G$ on $X$ to be transitive) and define

$$
s(x)=\varkappa_{\tau_{x}} . s\left(x^{\star}\right),
$$

which is contained in $Q \cap p^{-1}(x)$ due to (22) and (21).

In order to finish the proof of the lemma, it suffices to show $s(\pi . x)=\varkappa_{\pi} . s(x)$ for every $x \in X$ and $\pi \in G$. To deduce this equation, observe that due to 21) we have

$$
\begin{aligned}
\varkappa_{\tau_{\pi, x}}^{-1} \varkappa_{\pi} \varkappa_{\tau_{x}} \cdot p^{-1}\left(x^{\star}\right)= & \varkappa_{\tau_{\pi \cdot x}}^{-1} \cdot\left(\varkappa_{\pi} \cdot\left(\varkappa_{\tau_{x}} \cdot p^{-1}\left(x^{\star}\right)\right)\right) \\
& =\varkappa_{\tau_{\pi \cdot x}}^{-1} \cdot\left(\varkappa_{\pi} \cdot p^{-1}(x)\right)=\varkappa_{\tau_{\pi \cdot x}}^{-1} \cdot p^{-1}(\pi \cdot x)=p^{-1}\left(x^{\star}\right) .
\end{aligned}
$$


Thus, $\varkappa=\varkappa_{\tau_{\pi . x}}^{-1} \varkappa_{\pi} \varkappa_{\tau_{x}} \in \tilde{S}\left(x^{\star}\right)$ holds, and in particular, $\sigma \mapsto \varkappa \sigma$ defines a bijection $\tilde{S}\left(x^{\star}\right) \rightarrow \tilde{S}\left(x^{\star}\right)$. Therefore, we can conclude

$$
\varkappa_{\tau_{\pi \cdot x}}^{-1} \varkappa_{\pi} \varkappa_{\tau_{x}} . s\left(x^{\star}\right)=\varkappa . s\left(x^{\star}\right)=\frac{1}{\left|\tilde{S}\left(x^{\star}\right)\right|} \sum_{\sigma \in \tilde{S}\left(x^{\star}\right)} \varkappa \sigma \cdot y^{\star}=s\left(x^{\star}\right)
$$

from 23, which implies the equation

$$
\varkappa_{\pi} \cdot s(x)=\varkappa_{\pi} \cdot\left(\varkappa_{\tau_{x}} . s\left(x^{\star}\right)\right)=\varkappa_{\pi} \varkappa_{\tau_{x}} . s\left(x^{\star}\right)=\varkappa_{\tau_{\pi \cdot x}} . s\left(x^{\star}\right)=s(\pi \cdot x)
$$

that we needed to establish.

If $s: X \rightarrow Q$ is a coordinate symmetric section, then $G$ acts on the set $\mathcal{S}=$ $\left\{s_{1}, \ldots, s_{d}\right\}$ of the component functions of $s$ via

$$
\pi \cdot s_{j}=s_{\varkappa_{\pi-1}^{-1}(j)}
$$

for each $j \in[d]$. In order to see that this definition indeed is well-defined (note that $s_{1}, \ldots, s_{d}$ need not be pairwise distinct functions) and yields a group action, observe that, for each $j \in[d]$ and $\pi \in G$, we have

$$
\left(\pi . s_{j}\right)(x)=s_{\varkappa_{\pi^{-1}}^{-1}(j)}(x)=\left(\varkappa_{\pi^{-1}} . s(x)\right)_{j}=s_{j}\left(\pi^{-1} \cdot x\right) \quad \text { for all } x \in X,
$$

from which one deduces $1 . s_{j}=s_{j}$ for the one-element 1 in $G$ as well as $\left(\pi \pi^{\prime}\right) . s_{j}=$ $\pi .\left(\pi^{\prime} . s_{j}\right)$ for all $\pi, \pi^{\prime} \in G$. The isotropy group of $s_{j} \in \mathcal{S}$ under this action is

$$
\operatorname{iso}_{G}\left(s_{j}\right)=\left\{\pi \in G: \pi . s_{j}=s_{j}\right\} .
$$

From (25) one deduces

$$
s_{j}(x)=s_{j}\left(\pi^{-1} \cdot x\right) \quad \text { for all } x \in X, \pi \in \operatorname{iso}_{G}\left(s_{j}\right) .
$$

In general, it will be impossible to identify the isotropy groups iso ${ }_{G}\left(s_{j}\right)$ without more knowledge on the section $s$. However, for each isotropy group iso ${ }_{G}\left(s_{j}\right)$, one can at least bound its index $\left(G: \operatorname{iso}_{G}\left(s_{j}\right)\right)=|G| /\left|\operatorname{iso}_{G}\left(s_{j}\right)\right|$ in $G$, which will allow us to identify (large) subgroups of $\operatorname{iso}_{G}\left(s_{j}\right)$ later.

Lemma 13. In the setting described above, we have $\left(G: \operatorname{iso}_{G}\left(s_{j}\right)\right) \leq d$.

Proof. This follows readily from the fact that the index $\left(G: \operatorname{iso}_{G}\left(s_{j}\right)\right)$ of the isotropy group of the element $s_{j} \in \mathcal{S}$ under the action of $G$ on $\mathcal{S}$ equals the cardinality of the orbit of $s_{j}$ under that action, which due to $|\mathcal{S}| \leq d$, clearly is bounded from above by $d$.

Finally, the following result will turn out to be useful in order to derive lower bounds on the sizes of symmetric extensions for one polytope from bounds for another one.

Lemma 14. Let $Q \subseteq \mathbb{R}^{d}$ be an extension of the polytope $P \subseteq \mathbb{R}^{m}$ with projection $p: \mathbb{R}^{d} \rightarrow \mathbb{R}^{m}$, and let the face $P^{\prime}$ of $P$ be an extension of a polytope $R \subseteq \mathbb{R}^{k}$ with projection $q: \mathbb{R}^{m} \rightarrow \mathbb{R}^{k}$. Then the face $Q^{\prime}=p^{-1}\left(P^{\prime}\right) \cap Q \subseteq \mathbb{R}^{d}$ of $Q$ is an extension of $R$ via the composed projection $q \circ p: \mathbb{R}^{d} \rightarrow \mathbb{R}^{k}$.

If the extension $Q$ of $P$ is symmetric with respect to a group $G$, and $H$ is a group of affine transformations such that for every $\tau \in H$ we have $\tau . R=R$ and there 
is some $\pi_{\tau} \in G$ with $\pi_{\tau} . P^{\prime}=P^{\prime}$ and $q\left(\pi_{\tau} . x\right)=\tau . q(x)$ for all $x \in P^{\prime}$, then the extension $Q^{\prime}$ of $R$ is symmetric (with respect to the action of the group $H$ ).

Proof. Due to $q\left(p\left(Q^{\prime}\right)\right)=q\left(P^{\prime}\right)=R$, the polyhedron $Q^{\prime}$ (together with the projection $q \circ p$ ) clearly is an extension of $R$. In order to prove the statement on the symmetry of this extension, let $\tau \in H$ be an arbitrary element of $H$ with $\pi_{\tau} \in G$ as guaranteed to exist for $\tau$ in the statement of the lemma, and let $\varkappa_{\pi_{\tau}} \in \mathcal{O}(d)$ satisfy $\varkappa_{\pi_{\tau}} \cdot Q=Q$ and $(8)$ (as guaranteed to exist by the symmetry of the extension $Q$ of $P$ ). Since, for all $y \in Q^{\prime}$, we obviously have

$$
q\left(p\left(\varkappa_{\pi_{\tau}} \cdot y\right)\right)=q\left(\pi_{\tau} \cdot p(y)\right)=\tau \cdot(q(p(y))),
$$

it suffices to show $\varkappa_{\pi_{\tau}} \cdot Q^{\prime}=Q^{\prime}$. As $y \mapsto \varkappa_{\pi_{\tau}}$.y defines an automorphism of $Q$ (mapping faces of $Q$ to faces of the same dimension), it suffices to show $\varkappa_{\pi_{\tau}} \cdot Q^{\prime} \subseteq$ $Q^{\prime}$. Due to $\varkappa_{\pi_{\tau}} \cdot Q=Q$ this relation is implied by $\varkappa_{\pi_{\tau}} \cdot p^{-1}\left(P^{\prime}\right) \subseteq p^{-1}\left(P^{\prime}\right)$, which finally follows from

$$
p\left(\varkappa_{\pi_{\tau}} \cdot p^{-1}\left(P^{\prime}\right)\right)=\pi_{\tau} \cdot p\left(p^{-1}\left(P^{\prime}\right)\right)=\pi_{\tau} \cdot P^{\prime}=P^{\prime} .
$$

\section{Bounds on SyMmetric EXTEnsions of $\mathrm{P}_{\text {match }}^{\ell}(n)$}

In this section, we prove the following result, where all crucial ideas are taken from Yannakakis' paper [18] (though here presented in a different way).

Theorem 15. For every $n \geq 3$ and odd $\ell$ with $\ell \leq \frac{n}{2}$, there exists no coordinatesymmetric subspace extension for $\mathrm{P}_{\text {match }}^{\ell}(n)$ with at most $\left(\begin{array}{c}n \\ (\ell-1) / 2\end{array}\right)$ variables (with respect to the group $\mathfrak{S}(n)$ acting via permuting the nodes of $K_{n}$ as described in the Introduction).

From Theorem 15, we can derive the following more general lower bounds. Since we need it in the proof of the next result, and also for later reference, we state a simple fact on binomial coefficients first.

Lemma 16. For each constant $b \in \mathbb{N}$ there is some constant $\beta>0$ with

$$
\left(\begin{array}{c}
M-b \\
N
\end{array}\right) \geq \beta\left(\begin{array}{c}
M \\
N
\end{array}\right)
$$

for all large enough $M \in \mathbb{N}$ and $N \leq \frac{M}{2}$.

Theorem 17. There is a constant $C>0$ such that, for all $n$ and $1 \leq \ell \leq \frac{n}{2}$, the size of every extension for $\mathrm{P}_{\text {match }}^{\ell}(n)$ that is symmetric (with respect to the group $\mathfrak{S}(n)$ acting via permuting the nodes of $K_{n}$ as described in the Introduction) is bounded from below by

$$
C \cdot\left(\begin{array}{c}
n \\
\lfloor(\ell-1) / 2\rfloor
\end{array}\right) \text {. }
$$

Proof. For odd $\ell$, this follows from Theorem 15 using Lemma 10 . For even $\ell$, the polytope $\mathrm{P}_{\text {match }}^{\ell-1}(n-2)$ is (isomorphic to) a face of $\mathrm{P}_{\text {match }}^{\ell}(n)$ defined by $x_{e}=1$ for an arbitrary edge $e$ of $K_{n}$. From this, as $\ell-1$ is odd (and not larger than 
$(n-2) / 2)$ with $\lfloor(\ell-2) / 2\rfloor=\lfloor(\ell-1) / 2\rfloor$, and due to Lemma 16 , the theorem follows by Lemma 14 .

For even $n$ and $\ell=n / 2$, Theorem 17 provides a similar bound to Yannakakis result (see Step 2 in the proof of [18, Theorem 1]) that no coordinate-symmetric subspace extension of the perfect matching polytope of $K_{n}$ has a number of variables that is bounded by $\left(\begin{array}{l}n \\ k\end{array}\right)$ for any $k<n / 4$.

Theorem 17] in particular implies that the size of every symmetric extension for $\mathrm{P}_{\text {match }}^{\ell}(n)$ with $\Omega(\log n) \leq \ell \leq n / 2$ is bounded from below by $n^{\Omega(\log n)}$, which has the following consequence (due to Lemma9).

Corollary 18. For $\Omega(\log n) \leq \ell \leq n / 2$, there is no compact extended formulation for $\mathrm{P}_{\text {match }}^{\ell}(n)$ that is symmetric (with respect to the group $G=\mathfrak{S}(n)$ acting via permuting the nodes of $K_{n}$ as described in the Introduction).

The rest of this section is devoted to prove Theorem 15 . Throughout, with $\ell=$ $2 k+1$, we assume that $Q \subseteq \mathbb{R}^{d}$ with $d \leq\left(\begin{array}{l}n \\ k\end{array}\right)$ is a coordinate-symmetric subspace extension of $\mathrm{P}_{\text {match }}^{2 k+1}(n)$ for $4 k+2 \leq n$. We will only consider the case $k \geq 1$, as for $\ell=1$ the theorem trivially is true (note that we restrict to $n \geq 3$ ). Coordinatesymmetry is meant with respect to the action of $G=\mathfrak{S}(n)$ on $\mathrm{P}_{\text {match }}^{2 k+1}(n)$ and on the set $X$ of vertices of $\mathrm{P}_{\text {match }}^{2 k+1}(n)$ as described in the introduction, and we assume $s: X \rightarrow Q$ to be a coordinate-symmetric section as guaranteed to exist by Lemma 12 . Thus, we have

$$
X=\left\{\chi(M) \in\{0,1\}^{E_{n}}: M \in \mathcal{M}^{2 k+1}(n)\right\},
$$

where $\mathcal{M}^{2 k+1}(n)$ is the set of all matchings $M \subseteq E_{n}$ with $|M|=2 k+1$ in the complete graph $K_{n}=\left(V, E_{n}\right)$ (with $V=[n]$ ), and

$$
(\pi \cdot \chi(M))_{\{v, w\}}=\chi(M)_{\left\{\pi^{-1}(v), \pi^{-1}(w)\right\}}
$$

holds for all $\pi \in \mathfrak{S}(n), M \in \mathcal{M}^{2 k+1}(n)$, and $\{v, w\} \in E_{n}$. In order to simplify notations, we will sometimes identify matchings with their characteristic vectors, e.g., we write $s(M)$ instead of $s(\chi(M))$ for $M \in \mathcal{M}^{2 k+1}(n)$, and we consider the action of $\mathfrak{S}(n)$ on $\mathcal{M}^{2 k+1}(n)$.

The proof will proceed by constructing a solution $\lambda \in \mathbb{R}^{\mathcal{M}^{2 k+1}(n)}$ to the system (6), (7) with respect to the inequality $x\left(E\left(V_{\star}\right)\right) \leq k$ (valid for $\mathrm{P}_{\text {match }}^{2 k+1}(n)$ ) for some arbitrarily chosen subset $V_{\star} \subseteq V$ of $\left|V_{\star}\right|=2 k+1$ nodes.

In order to determine such a $\lambda \in \mathbb{R}^{\mathcal{M}^{2 k+1}(n)}$, we choose an arbitrary subset $V^{\star} \subseteq V \backslash V_{\star}$ of cardinality $\left|V^{\star}\right|=\left|V_{\star}\right|=2 k+1$ disjoint from $V_{\star}$ and denote for all $i \in[2 k+1]_{\text {odd }}=\{1,3,5, \ldots, 2 k+1\}$

$$
\mathcal{M}_{i}^{\star}=\left\{M \in \mathcal{M}^{2 k+1}(n): M \subseteq E\left(V_{\star} \cup V^{\star}\right),\left|M \cap\left(V_{\star}: V^{\star}\right)\right|=i\right\},
$$

as well as $\mathcal{M}^{\star}=\mathcal{M}_{1}^{\star} \cup \mathcal{M}_{3}^{\star} \cup \cdots \cup \mathcal{M}_{2 k+1}^{\star}$ (the set of all perfect matchings on the $4 k+2$ nodes in $\left.V_{\star} \cup V^{\star}\right)$. In fact, we will construct a vector $\lambda \in \mathbb{R}^{\mathcal{M}^{2 k+1}(n)}$ with

$$
\lambda_{M}= \begin{cases}\lambda_{i} & \text { if } M \in \mathcal{M}_{i}^{\star} \\ 0 & \text { if } M \notin \mathcal{M}^{\star}\end{cases}
$$


for some values $\lambda_{1}, \lambda_{3}, \ldots, \lambda_{2 k+1} \in \mathbb{R}$ to be determined.

The equation (7) to be satisfied now easily reads

$$
\sum_{i \in[2 k+1]_{\mathrm{odd}}} \frac{i-1}{2} \cdot\left|\mathcal{M}_{i}^{\star}\right| \cdot \lambda_{i}=-1
$$

while (6), for the time being, remains

$$
\sum_{i \in[2 k+1]_{\text {odd }}} \sum_{M \in \mathcal{M}_{i}^{*}} s_{j}(M) \cdot \lambda_{i} \geq 0 \quad \text { for all } j \in[d] .
$$

We are now going to simplify $(28)$ by means of 26 by identifying suitable (large) subgroups of $\operatorname{iso}_{G}\left(s_{j}\right)$. Here, the crucial ingredient will be a result (formulated in Lemma 20p on subgroups of the symmetric group $\mathfrak{S}(n)$, where $\mathfrak{A}(n) \subseteq$ $\mathfrak{S}(n)$ is the alternating group formed by all even permutations of $[n]$. This result is Claim 2 in the proof of Thm. 1 of Yannakakis paper [18]. His proof (which we work out below in order to make the presentation self contained at this crucial point) relies on a theorem of Bochert's [3] stating that any subgroup $U$ of $\mathfrak{S}(m)$ that acts primitively on $[\mathrm{m}]$ (i.e. the action is transitive and there is no $Y \subseteq[\mathrm{m}]$ with $1<|Y|<m$ for which $Y \cap \sigma . Y \in\{Y, \varnothing\}$ holds for all $\sigma \in U)$ contains $\mathfrak{A}(m)$ or has index at least $\lfloor(m+1) / 2\rfloor$ !.

In the proof of Lemma 20, we use the following estimate on products of binomial coefficients.

Lemma 19. For all positive integer numbers $a, b, c_{1}, \ldots, c_{r} \in \mathbb{N} \backslash\{0\}$ with

$$
\max \left\{c_{1}, \ldots, c_{r}\right\} \leq \max \{a, b\} \quad \text { and } \quad \sum_{i=1}^{r}\left(c_{i}-1\right) \leq a+b-2,
$$

we have

$$
\prod_{i=1}^{r} c_{i} ! \leq a ! \cdot b !
$$

If any of the inequalities in (29) additionally is strict, then (30) is strict as well.

Proof. Let $2=x_{1} \leq x_{2} \leq \cdots \leq x_{p}$ and $2=y_{1} \leq y_{2} \leq \cdots \leq y_{q}$ be the ordered sequences of non-trivial factors (appearing with their multiplicities) on the left- and right-hand-side, respectively, of (30) (with $p=\sum_{i=1}^{r}\left(c_{i}-1\right)$ and $q=a+b-2$ ). Clearly, the two sequences are of the form $(1, \ldots, 1,2, \ldots, 2, \ldots, d \ldots d)$ with $d=\max \left\{c_{1}, \ldots, c_{r}\right\}$ for the $x$ - and $d=\max \{a, b\}$ for the $y$-sequence, as well as non-increasing multiplicities from $\{1, \ldots, r\}$ and $\{1,2\}$, respectively. Due to the second inequality in (29), we have $p \leq q$.

If $x_{i} \leq y_{i}$ holds for all $i \in[p]$, the statements to prove clearly are true. Otherwise, defining $i^{\star}=\min \left\{i \in[p]: x_{i}>y_{i}\right\}-1$ ( $\infty$ if the set is empty), we find that the multiplicity of each $x_{i}$ with $i>i^{\star}$ must be one (as the multiplicities are nonincreasing and the multiplicities of $y_{1}, \ldots, y_{i^{\star}}$ are at most two). The left-hand-side of (30) thus equals

$$
x_{1} x_{2} \cdots x_{i^{\star}} \cdot\left(x_{i^{\star}}+1\right)\left(x_{i^{\star}}+2\right) \cdots x_{p},
$$


and the right-hand-side of $(30)$ is at least

$$
y_{1} y_{2} \cdots y_{i^{\star}} \cdot\left(y_{i^{\star}}+1\right)\left(y_{i^{\star}}+2\right) \cdots \max \{a, b\}
$$

This proves the statements of the lemma, as $x_{p} \leq \max \{a, b\}$ holds, and, in case of $i^{\star}<p$, we have $x_{i^{\star}}=y_{i^{\star}}$.

Lemma 20. For each subgroup $U$ of $\mathfrak{S}(n)$ with $(\mathfrak{S}(n): U) \leq\left(\begin{array}{l}n \\ k\end{array}\right)$ for $1 \leq k<\frac{n}{4}$, there is some $W \subseteq[n]$ with $|W| \leq k$ such that

$$
\{\pi \in \mathfrak{A}(n): \pi(v)=v \text { for all } v \in W\} \subseteq U
$$

holds.

Proof. Let $1 \leq k<\frac{n}{4}$ and $U$ be a subgroup of $\mathfrak{S}(n)$ with $(\mathfrak{S}(n): U) \leq\left(\begin{array}{l}n \\ k\end{array}\right)$, i.e.,

$$
|U| \geq k ! \cdot(n-k) ! \text {. }
$$

Under the action of $U$ on the set $[n]$, there is some orbit $B \subseteq[n]$ of size $|B| \geq$ $n-k$. Indeed, this follows from (32) and Lemma 19, as, for the partitioning $[n]=B_{1} \cup \cdots \cup B_{q}$ of $[n]$ into orbits, we have $\left|B_{1}\right| ! \cdots\left|B_{q}\right| ! \geq|U|$. We will show that $W=[n] \backslash B$ (with $|W| \leq k$ ) satisfies (31).

Every $\pi \in \mathfrak{S}(n)$ induces two permutations $\pi_{W} \in \mathfrak{S}(W)$ and $\pi_{B} \in \mathfrak{S}(B)$ (where we denote by $\mathfrak{S}(L)$ and $\mathfrak{A}(L)$ the set of all, respectively all even, permutations of a subset $L \subseteq[n]$ ). With the group homomorphisms $\varphi_{B}: U \rightarrow \mathfrak{S}(B)$ and $\varphi_{W}: U \rightarrow \mathfrak{S}(W)$ defined via $\varphi_{B}(\pi)=\pi_{B}$ and $\varphi_{W}(\pi)=\pi_{W}$ for all $\pi \in U$, we define

$$
F=\varphi_{B}\left(\operatorname{ker}\left(\varphi_{W}\right)\right) .
$$

It suffices to show $\mathfrak{A}(B) \subseteq F$, which in turn follows by the above mentioned theorem of Bochert's [3] (see, e.g., [17, Thm. 14.2]), by establishing the following two statements:

(1) $F$ acts primitively on $B$.

(2) $(\mathfrak{S}(B): F)<\left\lfloor\frac{|B|+1}{2}\right\rfloor$ !

In order to show (1), we first show that $\operatorname{ker}\left(\varphi_{W}\right)$ (and thus its isomorphic image $F$ ) acts transitively on $B$. For this, we use the following fact (see, e.g., [17, Prop. 7.1]): If an action of a group $G$ is primitive, then the induced action of every normal subgroup $N$ of $G$ with $|N|>1$ is transitive. Choosing $G=U$ and $N=\operatorname{ker}\left(\varphi_{W}\right)$, we find that $G=U$ acts primitively on $B$, since, clearly, the action of $U$ on the orbit $B$ is transitive, and a non-trivial decompostion of $B$ into blocks $B=$ $B_{1} \cup \cdots \cup B_{r}$ of imprimitivity (i.e., for each $\pi \in G$, we have $\pi . B_{i}=B_{i}$ or $\pi . B_{i} \cap B_{i}=\varnothing$ ) with $r \geq 2$ and $\left|B_{1}\right|=\cdots=\left|B_{r}\right|=b \geq 2$ would imply $r ! \cdot(b !)^{r} \geq|U| \geq k ! \cdot(n-k)$ !, contradicting Lemma 19 (due to $r, b \leq \frac{|B|}{2} \leq$ $\left.\frac{n}{2}<n-k\right)$. As $\operatorname{ker}\left(\varphi_{W}\right)$ is normal in $U$ and we have $k ! \cdot(n-k) ! \leq|U|=$ $\left|\operatorname{im}\left(\varphi_{W}\right)\right| \cdot\left|\operatorname{ker}\left(\varphi_{W}\right)\right|$ with $\left|\operatorname{im}\left(\varphi_{W}\right)\right| \leq|W| ! \leq k$ !, we have $\left|\operatorname{ker}\left(\varphi_{W}\right)\right|>1$. Thus $F$ acts transitively on $B$. Similarly to the argument used above, a non-trivial decomposition of $B$ into blocks of imprimitivity under the action of $F$ would yield $r ! \cdot(b !)^{r} \geq|F|$ with $r, b \leq \frac{n}{2}<n-k$, thus $r ! \cdot(b !)^{r} \cdot|W| ! \geq|U| \geq k ! \cdot(n-k) !$ (with $|W| \leq k<n-k$ ), again contradicting Lemma 19. Hence (1) is established. 
Hence, it remains to prove (2). From $|U|=|F| \cdot\left|\operatorname{im}\left(\varphi_{W}\right)\right|$ we deduce $|F| \geq$ $(n-k)$ ! via (32) and $|W| \leq k$ !. Thus, it suffices to show

$$
|B|(|B|-1) \cdots(n-k+1)<\left\lfloor\frac{|B|+1}{2}\right\rfloor ! .
$$

Obviously, it suffices to establish this equation for the maximal possible cardinality $|B|=n$ and the maximal $k$ with $k<\frac{n}{4}$. Therefore, we have to prove

$$
n(n-1) \cdots(n-k+1)<\left\lfloor\frac{n+1}{2}\right\rfloor !
$$

with $n=4 q+r$ (for $q, r \in \mathbb{N}, r<4$ ) and

$$
k=\left\{\begin{array}{ll}
q & \text { if } r \neq 0 \\
q-1 & \text { if } r=0
\end{array} .\right.
$$

In both cases, we have $2 k<\left\lfloor\frac{n+1}{2}\right\rfloor=\left\lfloor 2 q+\frac{r+1}{2}\right\rfloor$, thus the right-hand-side of (33) has at least twice as many non-trivial factors as the left-hand-side, which, due to $n \leq 2\left\lfloor\frac{n+1}{2}\right\rfloor \leq 3\left(\left\lfloor\frac{n+1}{2}\right\rfloor-1\right) \leq \ldots$ (as long as the first factor does not exceed the second one) establishes (33).

Having established Lemma 20, we can now continue with the proof of Theorem 15. As we assumed $d \leq\left(\begin{array}{l}n \\ k\end{array}\right)$ (with $k<\frac{n}{4}$ due to $4 k+2 \leq n$ ), Lemmas 13 and 20 imply that, for each $j \in[d]$, there is some subset $V_{j} \subseteq V$ of nodes with $\left|V_{j}\right| \leq k$ and

$$
H_{j}=\left\{\pi \in \mathfrak{A}(n): \pi(v)=v \text { for all } v \in V_{j}\right\} \subseteq \operatorname{iso}_{\mathfrak{S}(n)}\left(s_{j}\right) .
$$

Two matchings $M, M^{\prime} \in \mathcal{M}^{2 k+1}(n)$ are in the same orbit under the action of the group $H_{j}$ if and only if we have

$$
M \cap E\left(V_{j}\right)=M^{\prime} \cap E\left(V_{j}\right) \quad \text { and } \quad V_{j} \backslash V(M)=V_{j} \backslash V\left(M^{\prime}\right) .
$$

Indeed, it is clear that (34) holds if we have $M^{\prime}=\pi \cdot M$ for some permutation $\pi \in$ $H_{j}$. In turn, if (34) holds, then there clearly is some permutation $\pi \in \mathfrak{S}(n)$ with $\pi(v)=v$ for all $v \in V_{j}$ and $M^{\prime}=\pi . M$. Due to $|M|=2 k+1>\left|V_{j}\right|$ there is some edge $\{u, w\} \in M$ with $u, w \notin V_{j}$. Denoting by $\tau \in \mathfrak{S}(n)$ the transposition of $u$ and $w$, we thus also have $\pi \tau(v)=v$ for all $v \in V_{j}$ and $M^{\prime}=\pi \tau$. $M$. As one of the permutations $\pi$ and $\pi \tau$ is even, say $\pi^{\prime}$, we find $\pi^{\prime} \in H_{j}$ and $M^{\prime}=\pi^{\prime} . M$, proving that $M$ and $M^{\prime}$ are contained in the same orbit under the action of $H_{j}$.

Together with (26), the characterization of the orbits of $H_{j}$ via (34) yields that we have the implication

$$
M \cap E\left(V_{j}\right)=M^{\prime} \cap E\left(V_{j}\right) \quad \Rightarrow \quad s_{j}(M)=s_{j}\left(M^{\prime}\right)
$$

for all $j \in[d]$ and $M, M^{\prime} \in \mathcal{M}^{\star}$ (note that we have $V(M)=V_{\star} \cup V^{\star}$ for all $\left.M \in \mathcal{M}^{\star}\right)$. Denoting by $\mathcal{A}_{j}$ the set of all matchings $A$ on $V_{\star} \cup V^{\star}$ with $A \cap E\left(V_{\star} \cup V^{\star} \backslash V_{j}\right)=\varnothing$ (thus, $\left.|A| \leq\left|V_{j}\right| \leq k\right)$ and $V_{j} \subseteq V(A)$ (thus $s_{j}(M)=$ $s_{j}\left(M^{\prime}\right)$ for all $M, M^{\prime} \in \mathcal{M}^{\star}$ with $A \subseteq M$ and $A \subseteq M^{\prime}$ ), we hence find values $s_{j}(A)$ for all $A \in \mathcal{A}_{j}$ such that 28) becomes

$$
\sum_{i \in[2 k+1]_{\text {odd }}} \sum_{A \in \mathcal{A}_{j}} s_{j}(A) \cdot\left|\left\{M \in \mathcal{M}_{i}^{\star}: A \subseteq M\right\}\right| \cdot \lambda_{i} \geq 0 .
$$


The crucial observation now is that, for each $A \in \mathcal{A}_{j}$,

$$
g_{A}(i)=\left|\left\{M \in \mathcal{M}_{i}^{\star}: A \subseteq M\right\}\right|
$$

can be written as $\left|\mathcal{M}_{i}^{\star}\right|$ times a polynomial of degree at most $|A| \leq k$. In order to see this, define

$$
a_{\star}=\left|A \cap E\left(V_{\star}\right)\right|, \quad a^{\star}=\left|A \cap E\left(V^{\star}\right)\right|, \quad a_{\star}^{\star}=\left|A \cap\left(V_{\star}: V^{\star}\right)\right|,
$$

and denote by $\overline{\mathcal{A}}$ the set of all matchings $A^{\prime} \subseteq E\left(V_{\star} \cup V^{\star}\right)$ with

$$
a_{\star}=\left|A^{\prime} \cap E\left(V_{\star}\right)\right|, \quad a^{\star}=\left|A^{\prime} \cap E\left(V^{\star}\right)\right|, \quad a_{\star}^{\star}=\left|A^{\prime} \cap\left(V_{\star}: V^{\star}\right)\right| .
$$

For symmetry reasons, we have

$$
\begin{aligned}
g_{A}(i) & =\frac{1}{|\overline{\mathcal{A}}|} \sum_{A^{\prime} \in \overline{\mathcal{A}}}\left|\left\{M \in \mathcal{M}_{i}^{\star}: A^{\prime} \subseteq M\right\}\right| \\
& =\frac{1}{|\overline{\mathcal{A}}|} \sum_{M \in \mathcal{M}_{i}^{\star}}\left|\left\{A^{\prime} \in \overline{\mathcal{A}}: A^{\prime} \subseteq M\right\}\right| \\
& =\left|\mathcal{M}_{i}^{\star}\right| \cdot \frac{1}{|\overline{\mathcal{A}}|}\left(\begin{array}{c}
(2 k+1-i) / 2 \\
a_{\star}
\end{array}\right) \cdot\left(\begin{array}{c}
i \\
a_{\star}^{\star}
\end{array}\right) \cdot\left(\begin{array}{c}
(2 k+1-i) / 2 \\
a^{\star}
\end{array}\right),
\end{aligned}
$$

where the product of the three binomial coefficients is a polynomial in $i$ of degree $a_{\star}+a_{\star}^{\star}+a^{\star}=|A| \leq k$.

Hence, the left-hand-side of (35) equals $s_{j}(A) \sum_{i \in[2 k+1]_{\text {odd }}} f_{j}(i) \cdot\left|\mathcal{M}_{i}^{\star}\right| \lambda_{i}$ with a polynomial $f_{j}(i)$ in $i$ of degree at most $k$ and $f_{j}(0)=0$ (note $a_{\star}^{\star} \geq 1$, thus $\left.\left(\begin{array}{c}0 \\ a_{\star}^{\star}\end{array}\right)=0\right)$. Since the left-hand-side of 27$]$ equals $\sum_{i \in[2 k+1]_{\text {odd }}} f_{0}(i) \cdot\left|\mathcal{M}_{i}^{\star}\right| \lambda_{i}$ with a polynomial $f_{0}(i)$ in $i$ of degree $1(\leq k)$ and $f_{0}(0)=-1$, the following lemma finally concludes the proof (by choosing $I=[2 k+1]_{\text {odd }}$ and $\lambda_{i}=\gamma_{i} /\left|\mathcal{M}_{i}^{\star}\right|$ ).

Lemma 21. For every subset $I \subseteq \mathbb{R}$ of cardinality $|I|=k+1$ there are numbers $\gamma_{i} \in \mathbb{R}(i \in I)$ such that

$$
\sum_{i \in I} f(i) \cdot \gamma_{i}=f(0)
$$

holds for all (univariate) polynomials $f$ of degree at most $k$.

Proof. Suppose $I=\left\{i_{1}, \ldots, i_{k+1}\right\}$ and set $i_{0}=0$ as well as $I_{0}=I \cup\left\{i_{0}\right\}$. We may assume $0 \notin I$, as otherwise the statement of the lemma is trivial. Since the vector space $\mathbb{R}^{\leq k}[t]$ of all (univariate) polynomials of degree at most $k$ has dimension $k+1$, the image of the linear map $\mathbb{R}^{\leq k}[t] \rightarrow \mathbb{R}^{I_{0}}$ defined via $f \mapsto$ $\left(f\left(i_{0}\right), \ldots, f\left(i_{k+1}\right)\right)$ is contained in a linear hyperplane $H \subseteq \mathbb{R}^{I_{0}}$. As $i_{0}, \ldots, i_{k+1}$ are pairwise different and every polynomial of degree at most $k$ is determined by its values at any choice of $k+1$ (pairwise different) arguments, $H$ does not contain any line parallel to a coordinate axis, and hence, the normal vectors to $H$ do not have any zero-entries. In particular, the hyperplane has a normal vector $\gamma \in \mathbb{R}^{I_{0}}$ whose $i_{0}$-coefficient equals -1 . 


\section{A NON-SYMMETRIC EXTENSION FOR $\mathrm{P}_{\text {match }}^{\ell}(n)$}

We shall establish the following result on the existence of extensions for cardinality restricted matching polytopes in this section.

Theorem 22. For all $n$ and $\ell$, there are extensions for $\mathrm{P}_{\text {match }}^{\ell}(n)$ whose sizes can be bounded by $2^{O(\ell)} n^{2} \log n$ (and for which the encoding lengths of the coefficients needed to describe them can be bounded by a constant).

In particular, Theorem 22 implies the following, although, according to Corollary 18 no compact symmetric extended formulations exist for $\mathrm{P}_{\text {match }}^{\ell}(n)$ with $\ell=\Theta(\log n)$.

Corollary 23. For all $n$ and $\ell \leq O(\log n)$, there are compact extended formulations for $\mathrm{P}_{\text {match }}^{\ell}(n)$.

The proof of Theorem 22 relies on the following result on the existence of small families of perfect-hash functions, which is from [1, Sect. 4].

Theorem 24 (Alon, Yuster, Zwick [1]). There are maps $\phi_{1}, \ldots, \phi_{q(n, r)}:[n] \rightarrow[r]$ with $q(n, r) \leq 2^{O(r)} \log n$ such that, for every $W \subseteq[n]$ with $|W|=r$, there is some $i \in[q(n, r)]$ for which the map $\phi_{i}$ is bijective on $W$.

Actually, based on results from [9, 16], Alon, Yuster, and Zwick even show that, given an index $i$ of one of the maps and an element $v \in[n]$, the value $\phi_{i}(v)$ can be computed in constant time (in the uniform cost model).

The mere extistence of such a family follows easily ${ }^{2}$ from observing that for $\phi$ chosen uniformaly at random from all maps $[n] \rightarrow[r]$ and for any $r$-element subset $W$ of $[n]$, the probability that $\phi$ is bijective on $W$ is $\frac{r !}{r^{r}}$. Choosing $m:=$ $\frac{r^{r+1}}{r !} \ln n$ such maps $\phi_{1}, \ldots, \phi_{m}$ independently, for every $r$-element subset $W$ of $[n]$ the probability that none of $\phi_{1}, \ldots, \phi_{m}$ is bijective on $W$ is

$$
\left(1-\frac{r !}{r^{r}}\right)^{m} \leq e^{-m \frac{r !}{r^{r}}} \leq n^{-r} .
$$

Thus, the probability that there is some $r$-element subset $W$ of $[n]$ on which none of the $\phi_{i}$ is bijective is at most

$$
\left(\begin{array}{l}
n \\
r
\end{array}\right) n^{-r}<1
$$

Hence, the probability that $\phi_{1}, \ldots, \phi_{m}$ have the desired property is non-zero. The proof is concluded by noting that we have $\frac{r^{r+1}}{r !}=2^{\Theta(r)}$ by Stirling's formula.

Additionally to Theorem 24, we will use the following construction of an extension of a polytope that is specified as the convex hull of some polytopes of which extensions are already available. The result essentially is due to Balas (see, e.g., [2, Thm.2.1]). In the form it is stated here, it is taken from [12, Cor. 3], where it is derived from general results on branched polyhedral systems. Actually, in this

\footnotetext{
${ }^{2}$ This was brought to our attention by the referees.
} 
section we will need only the special case that the extensions used in the construction are the polytopes themselves. However, we will face the slightly more general situation in our treatment of cycle polytopes in Section 5

Lemma 25. If the polytopes $P_{i} \subseteq \mathbb{R}^{m}$ (for $i \in[q]$ ) have extensions $Q_{i}$ of size $s_{i}$, respectively, then

$$
P=\operatorname{conv}\left(P_{1} \cup \cdots \cup P_{q}\right)
$$

has an extension of size $\sum_{i=1}^{q}\left(s_{i}+1\right)$.

In order to prove Theorem 22, let $\phi_{1}, \ldots, \phi_{q}$ be maps as guaranteed to exist by Theorem 24 with $r=2 \ell$ and $q=q(n, 2 \ell) \leq 2^{O(\ell)} \log n$, and denote $\mathcal{M}_{i}=$ $\left\{M \in \mathcal{M}^{\ell}(n): \phi_{i}\right.$ is bijective on $\left.V(M)\right\}$ for each $i \in[q]$. By Theorem 24, we have $\mathcal{M}^{\ell}(n)=\mathcal{M}_{1} \cup \cdots \cup \mathcal{M}_{q}$. Consequently,

$$
\mathrm{P}_{\text {match }}^{\ell}(n)=\operatorname{conv}\left(P_{1} \cup \cdots \cup P_{q}\right)
$$

with $P_{i}=\operatorname{conv}\left\{\chi(M): M \in \mathcal{M}_{i}\right\}$ for all $i \in[q]$. Using the concept of branched polyhedral systems mentioned above along with Edmonds' Matching Theorem [5] (see (5)), one finds (see [12, Sect. 4.4] for the derivation)

$$
\begin{aligned}
P_{i}=\left\{x \in \mathbb{R}_{+}^{E_{n}}: x_{E_{n} \backslash E^{i}}=\mathbf{0},\right. & x\left(\delta\left(\phi_{i}^{-1}(s)\right)\right)=1 \text { for all } s \in[2 \ell], \\
x\left(\delta\left(\phi_{i}^{-1}(S)\right)\right) & \geq 1 \text { for all } S \subseteq[2 \ell],|S| \text { odd }\},
\end{aligned}
$$

where $E^{i}=E_{n} \backslash \bigcup_{j \in[2 \ell]} E\left(\phi_{i}^{-1}(j)\right)$. As the sum of the number of variables and the number of inequalities in the description of $P_{i}$ is bounded by $2^{O(\ell)}+n^{2}$ (the summand $n^{2}$ coming from the nonnegativity constraints on $x \in \mathbb{R}_{+}^{E_{n}}$ and the constant in $O(\ell)$ being independent of $i$ ), we obtain an extension of $\mathrm{P}_{\text {match }}^{\ell}(n)$ of size $2^{O(\ell)} n^{2} \log n$ by Lemma 25 . This proves Theorem 22 .

\section{Extensions for CyCle Polytopes}

By a modification of Yannakakis' construction for the derivation of lower bounds on the sizes of symmetric extensions for traveling salesman polytopes from the corresponding lower bounds for matching polytopes [18, Thm. 2], we obtain lower bounds on the sizes of symmetric extensions for $\mathrm{P}_{\text {cycl }}^{\ell}(n)$. The lower bound $\ell \geq 42$ in the statement of the theorem is convenient with respect to both formulating the bound and proving its validity.

Theorem 26. There is a constant $C^{\prime}>0$ such that, for all $n$ and $42 \leq \ell \leq$ $n$, the size of every extension for $\mathrm{P}_{\mathrm{cycl}}^{\ell}(n)$ that is symmetric (with respect to the group $\mathfrak{S}(n)$ acting via permuting the nodes of $K_{n}$ as described in the Introduction) is bounded from below by

$$
C^{\prime} \cdot\left(\begin{array}{c}
\left\lfloor\frac{n}{3}\right\rfloor \\
\left\lfloor\left(\left\lfloor\frac{\ell}{6}\right\rfloor-1\right) / 2\right\rfloor
\end{array}\right) .
$$

Proof. For $\ell \leq n$, let us define $\bar{\ell} \in\{0, \ldots, 5\}$ and $n^{\prime}, \ell^{\prime} \in \mathbb{N}$ via

$$
\bar{\ell}=\ell \bmod 6, \quad n^{\prime}=\left\lfloor\frac{n-\bar{\ell}}{3}\right\rfloor, \quad \text { and } \quad \ell^{\prime}=\left\lfloor\frac{\ell}{6}\right\rfloor=\frac{\ell-\bar{\ell}}{6} .
$$


For later reference, let us argue that we have

$$
\ell^{\prime} \leq \frac{n^{\prime}}{2}
$$

In order to establish (37), we have to show

$$
\frac{\ell-\bar{\ell}}{3} \leq\left\lfloor\frac{n-\bar{\ell}}{3}\right\rfloor
$$

which follows readily for $\ell \leq n-2$ (due to $\lfloor a / 3\rfloor \geq(a-2) / 3$ for all $a \in \mathbb{Z}$ ). For $\ell \geq n-2$ (thus $0 \leq n-\ell \leq 2$ ) we have

$$
(n-\bar{\ell}) \bmod 3=((n-\bar{\ell}) \bmod 6) \bmod 3=(n-\ell) \bmod 3=n-\ell,
$$

and thus $(38)$ in this case is satisfied due to

$$
\left\lfloor\frac{n-\bar{\ell}}{3}\right\rfloor=\frac{n-\bar{\ell}}{3}-\frac{1}{3}((n-\bar{\ell}) \bmod 3)=\frac{n-\bar{\ell}}{3}-\frac{n-\ell}{3}=\frac{\ell-\bar{\ell}}{3} .
$$

As we have $3 n^{\prime}+\bar{\ell} \leq n$, we can find four pairwise disjoint subsets $S, T, R$, and $U$ of nodes of the complete graph $K_{n}=\left(V, E_{n}\right)$ on $n$ nodes with $|S|=|T|=$ $|U|=n^{\prime}$ and $|R|=\bar{\ell}$ (see Fig. 5). We denote the elements of these sets as follows:

$$
S=\left\{s_{1}, \ldots, s_{n^{\prime}}\right\} \quad T=\left\{t_{1}, \ldots, t_{n^{\prime}}\right\} \quad U=\left\{u_{1}, \ldots, u_{n^{\prime}}\right\} \quad R=\left\{r_{1}, \ldots, r_{\bar{\ell}}\right\}
$$

Define the subset

$$
E^{0}=(S: U) \cup(S: R) \cup\left\{\left\{t_{i}, v\right\} \in E_{n}: i \in\left[n^{\prime}\right], v \in V \backslash\left\{s_{i}, u_{i}\right\}\right\}
$$

of edges of $K_{n}$, and denote by $F$ the face of $\mathrm{P}_{\text {cycl }}^{\ell}(n)$ that is defined by $x_{e}=0$ for all $e \in E^{0}$.

Every cycle $C \in \mathcal{C}^{\ell}(n)$ with $C \cap E^{0}=\varnothing$ satisfies $|V(C) \cap T| \leq 2\lfloor\ell / 6\rfloor$, because $C$ visits at least two nodes (from $V \backslash T$ ) between any two visits to $T$, and $|V(C) \cap T|$ is even. Therefore, denoting

$$
\tilde{\mathcal{C}}=\left\{C \in \mathcal{C}^{\ell}(n): C \cap E^{0}=\varnothing,|V(C) \cap T|=2\lfloor\ell / 6\rfloor\right\},
$$

we find that

$$
\tilde{F}=\operatorname{conv}\{\chi(C): C \in \tilde{\mathcal{C}}\}=\{x \in F: x(\delta(T))=4\lfloor\ell / 6\rfloor\}
$$

is a face of $F$. Moreover, for every $C \in \tilde{\mathcal{C}}$, we have $|C \cap E(S)| \geq\lfloor\ell / 6\rfloor$. Thus, with

$$
\mathcal{C}^{\prime}=\{C \in \tilde{\mathcal{C}}:|C \cap E(S)|=\lfloor\ell / 6\rfloor\}
$$

we find that

$$
P^{\prime}=\operatorname{conv}\left\{\chi(C): C \in \mathcal{C}^{\prime}\right\}=\{x \in \tilde{F}: x(E(S))=\lfloor\ell / 6\rfloor\}
$$

is a face of $\tilde{F}$. It is the face

$$
P^{\prime}=\left\{x \in \mathrm{P}_{\text {cycl }}^{\ell}(n): x(E(S))=\lfloor\ell / 6\rfloor, x(\delta(T))=4\lfloor\ell / 6\rfloor, x_{E^{0}}=\mathbf{0}\right\}
$$

of $\mathrm{P}_{\mathrm{cycl}}^{\ell}(n)$. 


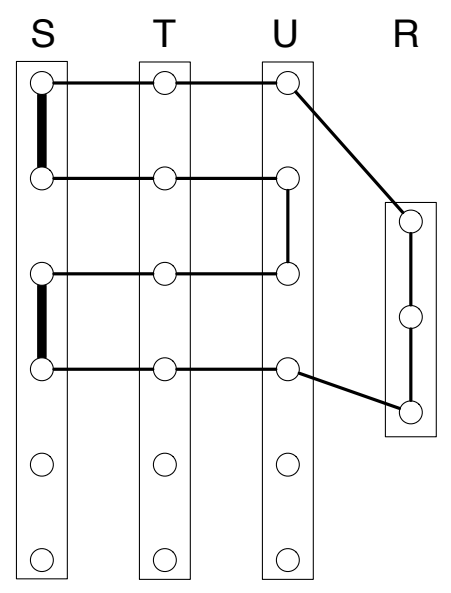

FIGURE 2. A cycle of length $\ell=15$ in $K_{21}$ inducing a matching of size 2 in $K_{5}$.

Since a cycle $C \in \mathcal{C}^{\ell}(n)$ is contained in $\mathcal{C}^{\prime}$ if and only if $C \cap E(S)$ is a matching of size $\ell^{\prime}=\lfloor\ell / 6\rfloor$, we find that via the orthogonal projection $q: \mathbb{R}^{E_{n}} \rightarrow \mathbb{R}^{E(S)}$ we have

$$
q\left(P^{\prime}\right)=\mathrm{P}_{\text {match }}^{\ell^{\prime}}\left(n^{\prime}\right)
$$

after identification of $S$ with the node set of $K_{n^{\prime}}$ via $s_{i} \mapsto i$ for all $i \in\left[n^{\prime}\right]$. Moreover, for every $\tau \in \mathfrak{S}\left(n^{\prime}\right)$ the permutation $\pi \in \mathfrak{S}(n)$ with

$$
\pi\left(s_{i}\right)=s_{\tau(i)}, \quad \pi\left(t_{i}\right)=t_{\tau(i)}, \quad \pi\left(u_{i}\right)=u_{\tau(i)}
$$

for all $i \in\left[n^{\prime}\right]$, and $\pi(r)=r$ for all $r \in R$ satisfies $\pi \cdot P^{\prime}=P^{\prime}$ and

$$
q(\pi . x)=\tau . q(x) \quad \text { for all } x \in \mathbb{R}^{E_{n^{\prime}}} .
$$

Hence, due to Lemma 14, a symmetric extension of $\mathrm{P}_{\text {cycl }}^{\ell}(n)$ of size $s$ yields a symmetric extension of $\mathrm{P}_{\text {match }}^{\ell^{\prime}}\left(n^{\prime}\right)$ of size at most $s+n^{2}$ (as one can define the face $P^{\prime}$ of $\mathrm{P}_{\text {cycl }}^{\ell}(n)$ by $2+\left|E^{0}\right| \leq n^{2}$ equations), which, due to 37) and Theorem 17 implies (with the constant $C>0$ from Theorem 17)

$$
s \geq \frac{C}{2} \cdot\left(\begin{array}{c}
\left\lfloor\frac{n-\bar{\ell}}{3}\right\rfloor \\
\left\lfloor\left(\left\lfloor\frac{\ell}{6}\right\rfloor-1\right) / 2\right\rfloor
\end{array}\right)
$$

for large enough $n$ (since, due to $\ell \geq 42$, the binomial coefficient in 39 ) grows at least cubically in $n$ ). Because of $\overline{\bar{\ell}} \leq 5$, Lemma 16 implies the existence of a constant $C^{\prime}>0$ as claimed in the theorem.

Corollary 27. For $\Omega(\log n) \leq \ell \leq n$, there is no compact extended formulation for $\mathrm{P}_{\text {cycl }}^{\ell}(n)$ that is symmetric (with respect to the group $\mathfrak{S}(n)$ acting via permuting the nodes of $K_{n}$ as described in the Introduction).

On the other hand, if we drop the symmetry requirement, we find extensions of the following size. 
Theorem 28. For all $n$ and $\ell$, there are extensions for $\mathrm{P}_{\text {cycl }}^{\ell}(n)$ whose sizes can be bounded by $2^{O(\ell)} n^{3} \log n$ (and for which the encoding lengths of the coefficients needed to describe them can be bounded by a constant).

Before we prove Theorem 28, we state a consequence that is similar to Corollary 18 for matching polytopes. It shows that, despite the non-existence of symmetric extensions for the polytopes associated with cycles of length $\Theta(\log n)$ (Corollary 27), there are non-symmetric compact extensions of these polytopes.

Corollary 29. For all $n$ and $\ell \leq O(\log n)$, there are compact extended formulations for $\mathrm{P}_{\mathrm{cycl}}^{\ell}(n)$.

The rest of the section is devoted to prove Theorem 28 , i.e., to construct an extension of $\mathrm{P}_{\text {cycl }}^{\ell}(n)$ whose size is bounded by $2^{O(\ell)} n^{3} \log n$. We proceed similarly to the proof of Theorem 22 (the construction of extensions for matching polytopes), this time starting with maps $\phi_{1}, \ldots, \phi_{q}$ as guaranteed to exist by Theorem 24 with $r=\ell$ and $q=q(n, \ell) \leq 2^{O(\ell)} \log n$, and defining

$$
\mathcal{C}_{i}=\left\{C \in \mathcal{C}^{\ell}(n): \phi_{i} \text { is bijective on } V(C)\right\}
$$

for each $i \in[q]$. Thus, we have $\mathcal{C}^{\ell}(n)=\mathcal{C}_{1} \cup \cdots \cup \mathcal{C}_{q}$, and hence,

$$
\mathrm{P}_{\text {cycl }}^{\ell}(n)=\operatorname{conv}\left(P_{1} \cup \cdots \cup P_{q}\right)
$$

with $P_{i}=\operatorname{conv}\left\{\chi(C): C \in \mathcal{C}_{i}\right\}$ for all $i \in[q]$. Due to Lemma 25, it suffices to exhibit, for each $i \in[q]$, an extension of $P_{i}$ of size bounded by $O\left(2^{\ell} \cdot n^{3}\right)$ (with the constant independent of $i$ ). Towards this end, let for $i \in[q]$

$$
V_{c}=\phi_{i}^{-1}(c) \text { for all } c \in[\ell],
$$

and define, for each $v^{\star} \in V_{\ell}$,

$$
P_{i}\left(v^{\star}\right)=\operatorname{conv}\left\{\chi(C): C \in \mathcal{C}_{i}, v^{\star} \in V(C)\right\} .
$$

Thus, we have

$$
P_{i}=\operatorname{conv} \bigcup_{v^{\star} \in V_{\ell}} P_{i}\left(v^{\star}\right),
$$

and hence, again due to Lemma 25, it suffices to construct extensions of the $P_{i}\left(v^{\star}\right)$, whose sizes are bounded by $O\left(2^{\ell} \cdot n^{2}\right)$.

In order to derive such extensions define, for each $i \in[q]$ and $v^{\star} \in V_{\ell}$, a directed acyclic graph $D$ with nodes

$$
(A, v) \text { for all } A \subseteq[\ell-1] \text { and } v \in \phi_{i}^{-1}(A),
$$

as well as two additional nodes $s$ and $t$, and arcs

$$
\left(s,\left(\left\{\phi_{i}(v)\right\}, v\right)\right) \text { and }(([\ell-1], v), t)
$$

for all $v \in \phi_{i}^{-1}([\ell-1])$, as well as

$$
\left((A, v),\left(A \cup\left\{\phi_{i}(w)\right\}, w\right)\right)
$$

for all $A \subseteq[\ell-1], v \in \phi_{i}^{-1}(A)$, and $w \in \phi_{i}^{-1}([\ell-1] \backslash A)$. This is basically the dynamic programming digraph (using an idea going back to [10]) from the 
color-coding method for finding paths of prescribed lengths described in [1]. Each $s$ - $t$-path in $D$ corresponds to a cycle in $\mathcal{C}_{i}$ that visits $v^{\star}$, and each such cycle, in turn, corresponds to two $s$-t-paths in $D$ (one for each of the two directions of transversal).

Defining $Q_{i}\left(v^{\star}\right)$ as the convex hull of the characteristic vectors of all $s$-t-paths in $D$ in the arc space of $D$, we find that $P_{i}\left(v^{\star}\right)$ is the image of $\left.Q_{i}\left(v^{\star}\right)\right)$ under the projection whose component function corresponding to the edge $\{v, w\}$ of $K_{n}$ is given by the sum of all arc variables corresponding to $\operatorname{arcs}\left((A, v),\left(A^{\prime}, w\right)\right)$ (for $A, A^{\prime} \subseteq[\ell-1]$ ) if $v^{\star} \notin\{v, w\}$, and by the sum of the two arc variables corresponding to $\left(s,\left(\left\{\phi_{i}(w)\right\}, w\right)\right)$ and $(([\ell-1], w), t)$ in case of $v=v^{\star}$. Clearly, $Q_{i}\left(v^{\star}\right)$ can be described by the nonnegativity constraints, the flow conservation constraints for all nodes in $D$ different from $s$ and $t$, and by the equation stating that there must be exactly one flow-unit leaving $s$. As the number of arcs of $D$ is bounded by $O\left(2^{\ell} \cdot n^{2}\right)$, we thus have found an extension of $P_{i}\left(v^{\star}\right)$ of the desired size.

\section{CONCLUSiOnS}

The results presented in this paper demonstrate that there are polytopes which have compact extended formulations though they do not admit compact symmetric ones. These polytopes are associated with matchings (or cycles) of some prescribed cardinalities. Nevertheless, whether there are compact extended formulations for general matching polytopes (or for perfect matching polytopes) or not, remains one of the most interesting open question here. In fact, it is even unknown whether there are any (non-symmetric) extended formulations of these polytopes of size $2^{\mathrm{o}(n)}$. In general, it is not at all well understood how small extended formulations of concrete polytopes can be. One problem is that the currently available methods to bound the sizes of general extended formulations from below have rather limited power (see [7]). Note, however, that via counting arguments one can prove, e.g., that there are 0/1-polytopes (even independence polytopes of matroids) that do not admit compact extended formulations [15].

In any case, the investigation of the limits of the concept of extended formulations seems to be not only a quite relevant topic from the point of view of optimization, but it also opens many interesting connections to other branches of mathematics. Some of these have played a role in this paper, others would be the nonnegative rank of matrices and communication complexity. For details, we refer once more to Yannakakis paper [18] (see also [7, 11]).

\section{REFERENCES}

[1] Noga Alon, Raphael Yuster, and Uri Zwick. Color-coding. J. Assoc. Comput. Mach., 42(4):844-856, 1995.

[2] Egon Balas. Disjunctive programming and a hierarchy of relaxations for discrete optimization problems. SIAM J. Algebraic Discrete Methods, 6(3):466-486, 1985.

[3] Alfred Bochert. Ueber die Zahl der verschiedenen Werthe, die eine Function gegebener Buchstaben durch Vertauschung derselben erlangen kann. Math. Ann., 33(4):584-590, 1889. 
[4] Michele Conforti, Gérard Cornuéjols, and Giacomo Zambelli. Extended formulations in combinatorial optimization. 4OR, 8(1):1-48, 2010.

[5] Jack Edmonds. Maximum matching and a polyhedron with 0, 1-vertices. J. Res. Nat. Bur. Standards Sect. B, 69B:125-130, 1965.

[6] Jack Edmonds. Matroids and the greedy algorithm. Math. Programming, 1:127-136, 1971.

[7] Samuel Fiorini, Volker Kaibel, K. Pashkovich, and Dirk Oliver. Theis. Combinatorial Bounds on Nonnegative Rank and Extended Formulations. ArXiv e-prints, November 2011, arXiv:1111.0444

[8] Samuel Fiorini, Serge Massar, Sebastian Pokutta, Hans Raj Tiwary, and Ronald de Wolf. Linear vs. semidefinite extended formulations: exponential separation and strong lower bounds. In STOC, pages 95-106, 2012.

[9] Michael L. Fredman, János Komlós, and Endre Szemerédi. Storing a sparse table with $O(1)$ worst case access time. J. Assoc. Comput. Mach., 31(3):538-544, 1984.

[10] Michael Held and Richard M. Karp. A dynamic programming approach to sequencing problems. J. Soc. Indust. Appl. Math., 10:196-210, 1962.

[11] Volker Kaibel. Extended formulations in combinatorial optimization. Optima, 85:2-7, 2011.

[12] Volker Kaibel and Andreas Loos. Branched polyhedral systems. In Friedrich Eisenbrand and Bruce Shepherd, editors, Integer Programming and Combinatorial Optimization (Proc. IPCO XIV), volume 6080 of LNCS, pages 177-190. Springer, 2010.

[13] Volker Kaibel, Kanstantsin Pashkovich, and Dirk Oliver Theis. Symmetry matters for the sizes of extended formulations. In Friedrich Eisenbrand and Bruce Shepherd, editors, Integer Programming and Combinatorial Optimization (Proc. IPCO XIV), volume 6080 of LNCS, pages 135-148. Springer, 2010.

[14] R. Kipp Martin. Using separation algorithms to generate mixed integer model reformulations. Technical report, University of Chicago, 1987.

[15] Thomas Rothvoß. Some 0/1 polytopes need exponential size extended formulations. ArXiv eprints, April 2011, arXiv:1105.0036

[16] Jeanette P. Schmidt and Alan Siegel. The spatial complexity of oblivious $k$-probe hash functions. SIAM J. Comput., 19(5):775-786, 1990.

[17] Helmut Wielandt. Finite permutation groups. Translated from the German by R. Bercov. Academic Press, New York, 1964.

[18] Mihalis Yannakakis. Expressing combinatorial optimization problems by linear programs. $J$. Comput. System Sci., 43(3):441-466, 1991.

VOLKER KAIBEL

E-mail address: kaibel @ovgu.de

KAnSTANTSIN PAShKovich

E-mail address: pashkovi@imo.math.uni-magdeburg.de

DIRK OLIVER THEIS

E-mail address: dirk.theiseovgu.de 